TAIWANESE JOURNAL OF MATHEMATICS

Vol. 13, No. 3, pp. 1017-1029, June 2009

This paper is available online at http://www.tjm.nsysu.edu.tw/

\title{
THE STRUCTURE OF LEFT FILIAL ALGEBRAS OVER A FIELD
}

\author{
M. Filipowicz ${ }^{1}$ and E. R. Puczyłowski ${ }^{2}$
}

\begin{abstract}
The structure of left filial algebras over fields is studied. Roughly speaking, these are algebras in which the relation "being a left ideal" is transitive. It is shown that semiprime algebras are left filial if and only if they are strongly regular and that prime radical algebras are left filial if and only if they are $H$-algebras, i.e., algebras in which all subalgebras are ideals. In the general case structure theorems describing left filial algebras are obtained. They make it possible to get a complete classification of finite dimensional left filial algebras over some fields.
\end{abstract}

\section{INTRODUCTION}

Many studies concern rings in which some kind of subrings are ideals or left (right) ideals. Among those are for instance duo rings (i.e., rings in which one-sided ideals are ideals) or quasi-duo rings (i.e., rings in which maximal one-sided ideals are ideals). The best known is the structure of $H$-rings, i.e., rings in which all subrings are ideals. They were studied in a number of papers (cf. [9]) but still their classification is not complete. Much wider is the class of filial (left filial) rings, i.e., rings $R$ such that if $I$ is an ideal (left ideal) of $J$ and $J$ is an ideal (left ideal) of $R$, then $I$ is an ideal (left ideal) of $R$. These rings were extensively studied in many papers (cf. [1-5], [11-14]). As these (and some other) papers show the structure of such rings is very complicated and far from a complete description.

In [8] Liu studied $H$-algebras over a field, i.e., $F$-algebras whose all subalgebras are ideals. It turned out that they are much easier to classify than $H$-rings and Liu obtained for them quite satisfactory structure theorems. In that context one may

Received September 8, 2007, accepted October 20, 2007.

Communicated by Wen-Fong Ke.

2000 Mathematics Subject Classification: 16A45, 16D15, 16D25.

Key words and phrases: Filial algebra, Left filial algebra, $H$-algebra, Strongly regular algebra.

${ }^{1}$ Supported by Technical University of Białystok grant S/WI/1/07.

${ }^{2}$ Supported by Flemish-Polish bilateral agreement BIL2005/VUB/06. 
expect that the structure of filial and/or left filial algebras over a field is also simpler than the structure of filial and/or left filial rings. This hope support some results obtained in [4-6] showing that it concerns algebras over some fields, which are left filial as rings.

This paper concerns that subject, i.e., we study here associative left filial algebras over fields. In Section 2 we show that for semiprime algebras the classes of left (right) filial algebras and strongly regular algebras coincide and they are strictly contained in the class of filial algebras. For prime radical algebras, the classes of filial, left (right) filial and $H$-algebras coincide (Section 3 ). In the general case we obtain in Section 4 structure theorems describing left filial algebras almost completely. They make it possible to classify finite dimensional algebras of such type over fields over which some quadratic forms are classified, in particular over algebraically closed and finite fields. We also show that algebras which are filial and left filial are right filial and describe their structure in Section 5.

Throughout the paper $F$ denotes a field, algebra means associative $F$-algebra and ideal (left ideal, right ideal) of an algebra $A$ means $F$-ideal (left $F$-ideal, right $F$-ideal) of $A$. To denote that $I$ is an ideal (left ideal, right ideal) of an algebra $A$ we write $I \triangleleft A\left(I<_{l} A, I<_{r} A\right)$.

For a given $F$-algebra $A$ we denote by $A^{*}$ the $F$-algebra obtained from $A$ by adjoining identity. If $X$ is a subset of $A$, then $l_{A}(X)$ (respectively, $r_{A}(X)$ ) denotes the left (respectively, right) annihilator of $X$ in $A$.

An algebra $A$ is called filial (left filial, right filial) if $I \triangleleft J \triangleleft A$ (respectively, $I<_{l} J<_{l} A, I<_{r} J<_{r} A$ ) implies $I \triangleleft A$ (respectively, $I<_{l} A, I<_{r} A$ ).

We start with characterizations of filial and left filial algebras. They are very similar to those known for rings but we include their proofs for completeness.

For a given element $a$ of an algebra $A$, we denote by $(a)$ the ideal of $A$ generated by $a$.

Proposition 1.1. (cf. [4]). An algebra $A$ is

(i) filial if and only if for every $a \in A,(a)=(a)^{2}+F a$;

(ii) left filial if and only if for every $a \in A, A^{*} a=A^{*} a^{2}+F a$.

\section{Proof.}

(i) Note that $(a)^{2}+F a \triangleleft(a) \triangleleft A$. Hence if $A$ is a filial algebra, then $(a)^{2}+F a \triangleleft A$. Obviously $a \in(a)^{2}+F a \subseteq(a)$, so $(a)=(a)^{2}+F a$. Suppose now that for every $a \in A,(a)=(a)^{2}+F a$ and $J \triangleleft I \triangleleft A$. Take any $j \in J$. Then $(j)=(j)^{2}+F j=\left((j)^{2}+F j\right)^{2}+F j \subseteq(j)^{3}+F j \subseteq I j I+F j \subseteq J$. This shows that $J \triangleleft A$ and (i) is proved.

(ii) Note that for every $a \in A, A^{*} a^{2}+F a<_{l} A^{*} a<_{l} A$. Hence if $A$ is left filial $a \in A^{*} a^{2}+F a<_{l} A$ and we get that $A^{*} a^{2}+F a=A^{*} a$. Conversely, let 
$K<_{l} L<_{l} A$. Take any $k \in K$. Then $A^{*} k=A^{*} k^{2}+F k \subseteq L k+F k \subseteq K$. This shows that $K<_{l} A$ and we are done.

\section{Semiprime Algebras}

An algebra $A$ is called strongly regular if for every $a \in A$ there exists $x \in A$ such that $a=x a^{2}$, equivalently, $a \in A a^{2}$.

Now we quickly recall some properties of strongly regular and related algebras, which will be needed later. More information concerning such algebras one can find in [7].

It is clear that strongly regular algebras are reduced (i.e., they do not contain non-zero nilpotent elements) and it is not hard to check that if $A$ is a reduced algebra, then for arbitrary elements $a, x \in A, a=x a^{2}$ if and only if $a=a^{2} x$ if and only if $a=a x a$. In particular, an algebra is strongly regular if and only if it is reduced and von Neumann regular. An algebra is von Neumann regular if and only if every finitely generated left (equivalently, right) ideal is generated by an idempotent. In reduced rings idempotents are central. Consequently strongly regular rings are duo rings, i.e., their one-side ideals are ideals.

It is clear that ideals of strongly regular algebras are strongly regular algebras as well and they are idempotent. Now if $J \triangleleft K \triangleleft A$ and $J^{2}=J$, then $A J=A K J=J$ and $J A=J K A=J$, so $J \triangleleft A$. These in particular show that strongly regular algebras are filial and left (right) filial.

Now we will show that semiprime left filial algebras are strongly regular.

We start with the following simple lemma.

Lemma 2.1. If $A$ is a reduced algebra and for some $a, b \in A$ and a positive integer $n, a b^{n}=0$, then $a b=0$.

Proof. Let $k$ be the smallest positive integer such that $a b^{k}=0$. If $k \geq 2$, then $\left(b a b^{k-1}\right)^{2}=0$. Hence, since $A$ is reduced, $b a b^{k-1}=0$. Now $\left(a b^{k-1}\right)^{2}=0$, so $a b^{k-1}=0$, a contradiction. Thus $k=1$ and hence $a b=0$.

Theorem 2.2. Every left filial semiprime algebra $A$ is strongly regular.

Proof. By Proposition 1.1, if $a \in A$ and $a^{2}=0$, then $A^{*} a=F a$, so $\left(A^{*} a\right)^{2}=$ 0 . Hence, since $A$ is semiprime, $A^{*} a=0$. Consequently $A^{*}$ is a reduced algebra.

Applying Proposition 1.1 we get that for every $a \in A, A^{*} a^{2}=A^{*} a^{4}+F a^{2}$. Hence there exist $x \in A^{*}$ and $\alpha \in F$ such that $a^{3}=x a^{4}+\alpha a^{2}$. Now $\left(a-x a^{2}-\right.$ $\alpha) a^{2}=0$, so by Lemma 2.1, $\left(a-x a^{2}-\alpha\right) a=0$. This implies that if $\alpha \neq 0$, then $a \in A^{*} a^{2}$. If $\alpha=0$, then $\left(a-x a^{2}\right) a=0$, so $(1-x a) a^{2}=0$ and by Lemma 2.1, $(1-x a) a=0$. Thus in both cases $a \in A^{*} a^{2}=\left(A^{*} a\right) a \subseteq\left(A^{*} a^{2}\right) a \subseteq A a^{2}$ and we are done. 
The above results show in particular that semiprime algebras are left filial if and only if they are right filial (so they are filial). Obviously every simple algebra is filial but a strongly regular algebra is simple if and only if it is a division algebra. Thus there are many semiprime filial algebras which are not left filial.

Let us note that also semiprime rings are left filial if and only if they are right filial ([4]) but the example of the ring of integers shows that it is not true that they are strongly regular. The proof of that result for rings is much more complicated than for algebras.

\section{Prime Radical Algebras}

In this section we study the structure of prime radical algebras, which are filial or left filial. We start with the following

Theorem 3.1. If a prime radical algebra $A$ is filial or left filial, then $A^{3}=0$.

Proof. Suppose first that $A$ is nilpotent and let $k$ be the smallest positive integer such that $A^{k}=0$. If $k \geq 4$, then for every $a \in A^{k-2},(a)^{2}=0$. Hence by Proposition 1.1, $A^{*} a=F a$ when $A$ is a left filial algebra and $(a)=F a$ when $A$ is a filial algebra. In both cases, for every $x \in A$ there exists $\alpha \in F$ such that $x a=\alpha a$. However $x \in A$ is nilpotent, so if $0 \neq \alpha \in F$, then $\alpha-x$ is invertible in $A^{*}$ and $a=0$. Consequently for every $a \in A^{k-2}, A a=0$, so $A^{k-1}=A A^{k-2}=0$, which contradicts minimality of $k$. Therefore $k \leq 3$ and we are done.

Now let $A$ be an arbitrary prime radical filial or left filial algebra. Applying Zorn's lemma one easily notes that there exists an ideal $M$ maximal in the family of ideals $I$ of $A$ such that $I^{3}=0$. If $A \neq M$, then $A / M$, as a non-zero prime radical algebra, contains a non-zero nilpotent ideal $I / M$. Obviously $I$ is a nilpotent filial or left filial algebra. Hence by the foregoing, $I^{3}=0$. This contradiction shows that $A=M$, so $A^{3}=0$.

Corollary 3.2. A prime radical algebra is filial if and only if it is an H-algebra.

Proof. Suppose that $A$ is a prime radical filial algebra. By Theorem 3.1, $A^{3}=0$. Let $B$ be a subalgebra of $A$. Note that $B \triangleleft B+A^{2} \triangleleft A$. Hence, since $A$ is a filial algebra, $B \triangleleft A$. This shows that $A$ is an $H$-algebra. The opposite implication is clear.

The next theorem shows in particular that also the class of prime radical left filial algebras coincides with the class of prime radical $H$-algebras.

Theorem 3.3. For a given prime radical algebra $A$ the following conditions are equivalent 
(i) A is left filial;

(ii) $A^{3}=0$ and for every $a \in A, A a=F a^{2}$;

(iii) $A^{3}=0$ and for every $x \in A, A x=x A=F x^{2}$;

(iv) $A$ is an $H$-algebra.

Proof. (i) $\Rightarrow$ (ii). Theorem 3.1 shows that $A^{3}=0$ and from Proposition 1.1 we get that for every $a \in A, A^{*} a=A^{*} a^{2}+F a$. Hence $A^{*} a=F a+F a^{2}$. Take any $x \in A$. Then $x a=\alpha a+\beta a^{2}$ for some $\alpha, \beta \in F$. Now $x^{2} a=\alpha x a+\beta x a^{2}$ and, since $A^{3}=0, \alpha x a=0$. If $\alpha \neq 0$, then $x a=0$. If $\alpha=0$, then $x a=\beta a^{2}$. In both cases $x a \in F a^{2}$. Hence $A a=F a^{2}$ and (ii) follows.

(ii) $\Rightarrow$ (iii). It suffices to show that for every $y \in A, x y \in F x^{2}$. Applying (ii) to $a=y$ we get that $x y=\alpha y^{2}$ for some $\alpha \in F$. If $\alpha=0$, then obviously $x y \in F x^{2}$. Thus assume that $\alpha \neq 0$. Note that $(x-\alpha y)^{2}=x^{2}-\alpha y x$. Hence applying (ii) to $a=x-\alpha y$ and next to $a=x$ we get that $x(x-\alpha y) \in F\left(x^{2}-\alpha y x\right) \subseteq F x^{2}$. This obviously implies that $x y \in F x^{2}$.

(iii) $\Rightarrow$ (iv). Let $B$ be any subalgebra of $A$. From (iii) we immediately get that for every $b \in B, F b+F b^{2} \triangleleft A$. Obviously $B=\sum_{b \in B}\left(F b+B b^{2}\right)$. Hence $B \triangleleft A$ and (iv) follows.

The implication (iv) $\Rightarrow$ (i) is clear.

Remark 3.4. In [5] it was proved that also prime radical filial rings are $H$ rings. However the proof for rings is much harder than for algebras. The examples $n Z / n^{k} Z$, where $Z$ is the ring of integers, show that Theorem 3.1 does not hold for rings. Note also that there are left filial, nilpotent of index 3 rings, which are not filial ([5]). Obviously they are not $H$-rings.

The following result can be derived from the main theorem proved in [8]. We include its quick proof based on Theorem 3.3 (iii).

Corollary 3.5. A prime radical algebra $A$ is left filial if and only if $A^{2}=0$ or $A=B \oplus C$, where $C^{2}=0, B^{2}=F b$ for some $0 \neq b \in B$ with $B b=b B=0$ and for every $x \in B \backslash F b, x^{2} \neq 0$.

Proof. Suppose that $A$ is left filial and $x, y \in A$ are such that $x^{2} \neq 0 \neq y^{2}$. If $x y \neq 0$ or $y x \neq 0$, then by Theorem 3.3 (iii), $F x y+F y x=F x^{2}=F y^{2}$. If $x y=y x=0$, then $x^{2}=x(x+y) \in F(x+y)^{2}=F\left(x^{2}+y^{2}\right)$, so $x^{2}=\alpha\left(x^{2}+y^{2}\right)$ for some $\alpha \in F$ and $(1-\alpha) x^{2}=\alpha y^{2}$. This easily implies that again $F x^{2}=F y^{2}$. Consequently $A^{2}=0$ or there is $a \in A$ such that $A^{2}=F a^{2} \neq 0$. From Theorem 3.3 (iii) it also follows that $T=\left\{x \in A \mid x^{2}=0\right\}=\{x \in A \mid x A=A x=0\}$ and $b=a^{2} \in T$. Let $C$ be a maximal $F$-subspace of $T$, which does not contain $b$ and let $B$ be the $F$-linear complement of $C$ in $A$ containing $b$. The relations 
obtained above immediately show that $B, C$ and $b$ satisfy the conditions stated in the corollary.

The get the opposite implication it suffices to note that if $A$ is an algebra of the form described in the "if" part and $U$ is a subalgebra of $A$, then $b \in U$ or $A U=U A=0$. In both cases $U$ is an ideal of $A$.

As we have proved in Theorem 3.3 all prime radical left filial algebras are $H$ algebras. Thus the results concerning the structure of $H$-algebras obtained in [8] apply to prime radical left filial algebras. In particular from Corollary 2 proved in [8] it follows that if every polynomial over $F$ of degree 2 has a root in $F$, then a prime radical algebra $A$ is left filial if and only if $A^{2}=0$ or $A=B \oplus C$, where $B^{2}=0$ and $C \simeq x F[x] / x^{3} F[x]$. From results proved in [8] it also follows that for an arbitrary $F$ the classification problem of finite dimensional prime radical left filial algebras is equivalent to a linear algebra problem (roughly speaking concerning a classification of $F$-bilinear mappings).

We close this section with two results concerning a classification of indecomposable prime radical left filial algebras.

We say that an algebra is indecomposable if it is not isomorphic to a direct sum of two non-zero algebras.

The quadratic form $\sum_{1 \leq i, j \leq n} f_{i j} x_{i} x_{j}$ in indeterminates $x_{1}, \cdots, x_{n}$ over $F$ is called isotropic if there are $\alpha_{1}, \cdots, \alpha_{n} \in F$, not all equal 0 , such that $\sum_{1 \leq i, j \leq n}$ $f_{i j} \alpha_{i} \alpha_{j}=0$.

Proposition 3.6. If every quadratic form over $F$ in $n$ indeterminates is isotropic and $A$ is a prime radical left filial indecomposable algebra, then $\operatorname{dim}{ }_{F} A \leq n$.

Proof. If $A^{2}=0$, then, since $A$ is indecomposable, $\operatorname{dim}_{F} A \leq 1$. Thus assume that $A^{2} \neq 0$. Then by Corollary 3.5, there is $0 \neq a \in A$ such that $A^{2}=F a^{2} \neq 0$ and $x^{2} \neq 0$ for every $0 \neq x \in A \backslash F a^{2}$. Let $e_{1}=a, e_{2}, \cdots, e_{n} \in A$. For arbitrary $1 \leq i, j \leq n$ there is $f_{i j} \in F$ such that $e_{i} e_{j}=f_{i j} a^{2}$. By the assumption the quadratic form $\sum_{1 \leq i, j \leq n} f_{i j} x_{i} x_{j}$ is isotropic. Hence there are $\alpha_{1}, \cdots, \alpha_{n} \in F$, not all equal 0 , such that $\sum_{1 \leq i, j \leq n} f_{i j} \alpha_{i} \alpha_{j}=0$. Then $\left(\alpha_{1} e_{1}+\cdots+\alpha_{n} e_{n}\right)^{2}=0$. Hence $\alpha_{1} e_{1}+\cdots+\alpha_{n} e_{n} \in \bar{F} a^{2}$. This shows that $\operatorname{dim}_{F} A \leq n$.

It is known (cf. [10]) that if $F$ is a finite field, then the assumption of Proposition 3.6 is satisfied for $n=3$. Thus indecomposable prime radical left filial algebras over such fields are of dimension $\leq 3$.

It is clear that a non-zero prime radical indecomposable algebra is of dimension $\leq 2$ if and only if $A \simeq x F[x] / x^{2} F[x]$ or $A \simeq x F[x] / x^{3} F[x]$. In the following proposition we describe indecomposable prime radical left filial algebras of dimension 3. 
Proposition 3.7. An indecomposable prime radical algebra with $\operatorname{dim}_{F} A=3$ is left filial if and only if it contains a basis $\left\{e_{1}, e_{2}, e_{3}\right\}$ such that $e_{1}^{2}=e_{3}, e_{2}^{2}=\alpha e_{3}$, $e_{1} e_{2}=0, e_{2} e_{1}=\beta e_{3}$ and $e_{i} e_{3}=e_{3} e_{i}=0$ for all $1 \leq i \leq 3$, where $0 \neq \alpha \in F$ and $\beta \in F$ are such that the equation $1+\beta t+\alpha t^{2}=0$ has no solution in $F$.

Proof. Suppose that $A$ is an indecomposable prime radical left filial algebra with $\operatorname{dim}_{F} A=3$. By Corollary 3.5 there is $e_{1} \in A$ such that $e_{3}=e_{1}^{2} \neq 0$ and $A^{2}=F e_{3}$. Since $\operatorname{dim}_{F} A=3$, there exists $e_{2} \neq 0$ such that $e_{1} e_{2}=0$. It is clear that $e_{1}, e_{2}, e_{3}$ form a basis of $A$. Moreover since for every $x \in A \backslash F e_{3}$, $x^{2} \neq 0$, there is $0 \neq \alpha \in F$ such that $e_{2}^{2}=\alpha e_{3}$. Since $A^{2}=F e_{3}$, there is $\beta \in F$ such that $e_{2} e_{1}=\beta e_{3}$ and, since $A^{3}=0, e_{i} e_{3}=e_{3} e_{i}=0$ for all $1 \leq i \leq 3$. Let $f \in F$ and $x=e_{1}+f e_{2}$. Obviously $x \in A \backslash F e_{3}$. Hence $x^{2}=\left(1+\beta f+\alpha f^{2}\right) e_{3} \neq 0$. Consequently $1+\beta f+\alpha f^{2} \neq 0$ and hence the equation $1+\beta t+\alpha t^{2}=0$ has no solution in $F$. This proves the "only if" part of the proposition. It is not hard to see that, in view of Corollary 3.5, to get the "if" part it suffices to prove that for arbitrary $f_{1}, f_{2} \in F$, if $\left(f_{1} e_{1}+f_{2} e_{2}\right)^{2}=0$, then $f_{1}=f_{2}=0$. Now $\left(f_{1} e_{1}+f_{2} e_{2}\right)^{2}=f_{1}^{2}+f_{1} f_{2} \beta+f_{2}^{2} \alpha$, so if it is equal to 0 , then $f_{1}^{2}+\beta f_{1} f_{2}+\alpha f_{2}^{2}=0$. Note that if $f_{1}=0$, then, since $\alpha \neq 0, f_{2}=0$ and we are done. Otherwise the equation $1+\beta t+\alpha t^{2}=0$ would have a solution in $F$, a contradiction.

Applying Corollary 3.5, Proposition 3.6, Proposition 3.7 and the fact that over finite fields quadratic forms in 3 indeterminates are isotropic, we immediately obtain the following complete classification of prime radical left filial algebras over finite fields.

Corollary 3.8. A prime radical algebra over a finite field $F$ is left filial if and only if $A \simeq B \oplus C$, where $B$ is an algebra such that $B^{2}=0$ and $C=0$ or $C \simeq x F[x] / x^{3} F[x]$ or $C$ is isomorphic to the algebra described in Proposition 3.7.

\section{General Case}

In what follows we denote by $\mathcal{R}(A)$ the prime radical of the algebra $A$.

We start with two lemmas.

Lemma 4.1. If $A$ is a left filial algebra, then for every idempotent $e \in A$, $e \mathcal{R}(A)=0$ or $e a=a$ for all $a \in \mathcal{R}(A)$.

Proof. Take any $a \in \mathcal{R}(A)$. From Theorem 3.3 it follows that $a^{3}=0$ and $F a+F a^{2}<_{l} A$. Thus $e a=\alpha a+\beta a^{2}$ for some $\alpha, \beta \in F$. If $\alpha=0$, then $e a$ $=e(e a)=\beta(e a) a=\beta^{2} a^{3}=0$. If $\alpha \neq 0$ and $a^{2} \neq 0$, then $\alpha a+\beta a^{2}=e a=$ $e(e a)=e\left(\alpha a+\beta a^{2}\right)=\alpha\left(\alpha a+\beta a^{2}\right)+\beta\left(\alpha a+\beta a^{2}\right) a=\alpha^{2} a+2 \alpha \beta a^{2}$. Since 
$a^{2} \neq 0$ and $a^{3}=0, a$ and $a^{2}$ are linearly independent over $F$. Consequently $\alpha^{2}=\alpha$ and $\beta=2 \alpha \beta$. This gives that $\alpha=1$ and $\beta=0$. Hence $e a=a$. Finally, if $\alpha \neq 0$, $a \neq 0$ and $a^{2}=0$, then $e a=\alpha a$ and $e a=e(e a)=\alpha^{2} a$. Consequently $\alpha=\alpha^{2}$, so $\alpha=1$ and $e a=a$. Therefore in all cases $e a=a$ or $e a=0$.

Suppose now that there exists $0 \neq x \in \mathcal{R}(A)$ such that $e x=x$. Take any $a \in \mathcal{R}(A)$. By the forgoing $x+e a=e(x+a)=x+a$ or $x+e a=e(x+a)=0$. In the former case $e a=a$ and in the latter $e a=-x \neq 0$. Hence $e a=a$. Thus indeed $e \mathcal{R}(A)=0$ or $e a=a$ for all $a \in \mathcal{R}(A)$.

Lemma 4.2. If $A$ is a left filial algebra, then for every $x \in A$ there exist $y \in A$ and an idempotent $e \in A$ such that $x-x y x, y x-e, x-x e \in \mathcal{R}(A)$.

Proof. By Theorem 2.2, $A / \mathcal{R}(A)$ is strongly regular. Hence for every $x \in A$ there exists $y \in A$ such that $x-x y x \in \mathcal{R}(A)$. Moreover $y x+\mathcal{R}(A)$ is an idempotent of $A / \mathcal{R}(A)$. It can be lifted to an idempotent in $A$, i.e., there exists an idempotent $e \in A$ such that $y x-e \in \mathcal{R}(A)$. Finally $x-x e=x-x y x+x(y x-e) \in \mathcal{R}(A)$.

Now we are ready to describe the structure of left filial algebras.

Theorem 4.3. $A$ is a left filial algebra if and only if $A / \mathcal{R}(A)$ is strongly regular, $\mathcal{R}(A)$ is an $H$-algebra and

(i) $A=l_{A}(\mathcal{R}(A))+\mathcal{R}(A)$

or

(ii) $A=F e+l_{A}(\mathcal{R}(A))+\mathcal{R}(A)$, where $\mathcal{R}(A) \neq 0$ and $e$ is an idempotent of $A$ such that $e b=b$ for every $b \in \mathcal{R}(A)$.

Proof. Suppose that $A$ is a left filial algebra. If $\mathcal{R}(A)=0$, then obviously $A=l_{A}(\mathcal{R}(A))$ and (i) holds. Thus suppose that there is $0 \neq a \in \mathcal{R}(A)$. We can assume that $a^{2}=0$. Then by Theorem 3.3, $\mathcal{R}(A) \subseteq l_{A}(a)$. Obviously $F a<_{l} A$. Hence $l_{A}(a)=A$ or $\operatorname{codim}_{F} l_{A}(a)=1$. If the latter holds, then there is $x \in A \backslash l_{A}(a)$ such that $F x+l_{A}(a)=A$. Applying Lemma 4.2 we get that there exists an idempotent $e \in A$ such that $x-x e \in \mathcal{R}(A)$. Since $\mathcal{R}(A) a=0$ and $x a \neq 0$, we have $e a \neq 0$. Hence by Lemma 4.1, $e b=b$ for every $b \in \mathcal{R}(A)$.

To get the "only if" part of the result it remains to prove that $l_{A}(a) \subseteq l_{A}(\mathcal{R}(A))+$ $\mathcal{R}(A)$. Take any $x \in l_{A}(a)$. Lemma 4.2 implies that there exist $y \in A$ and an idempotent $e \in A$ such that $x-x y x \in \mathcal{R}(A)$ and $y x-e \in \mathcal{R}(A)$. Since $x a=0$ and $\mathcal{R}(A) a=0$, we get $e a=0$. Hence by Lemma 4.1, $e \mathcal{R}(A)=0$. Now $x=x-x y x+x y x-x e+x e$. Since $x-x y x \in \mathcal{R}(A), x y x-x e=x(y x-e) \in \mathcal{R}(A)$ and $x e \in l_{A}(a)$, we get that $x \in l_{A}(a)+\mathcal{R}(A)$. Hence the "only if" part is proved.

To prove the "if" part suppose that $A$ is of the described form and $K<_{l} L<_{l} A$. From Theorem 3.3 and the form of $A$ it immediately follows that $K \cap \mathcal{R}(A)<_{l} A$. Take any $k \in K$. Since $A / \mathcal{R}(A)$ is strongly regular there exist $x \in A$ and $b \in \mathcal{R}(A)$ 
such that $k-x k^{2}=b$. Note that $k-x k^{2} \in K$, so $b \in K \cap \mathcal{R}(A)$. Now $A x k^{2} \subseteq K$ and $A b \subseteq K$. Consequently $A k \subseteq K$ and the result is proved.

Now we will describe more precisely the structure of algebras satisfying Theorem 4.3 (ii).

Let $U$ be an algebra with identity, $T$ an algebra and $M$ a $U-T$-bimodule, which is unitary as the left $U$-module. The set $\left(\begin{array}{cc}U & M \\ 0 & T\end{array}\right)$ of matrices of the form $\left(\begin{array}{ll}u & m \\ 0 & t\end{array}\right)$, where $u \in U, m \in M$ and $t \in T$, is an algebra with respect to the obvious matrix operations.

Theorem 4.4. $A$ is an algebra satisfying Theorem 4.3 (ii) if and only if $A \simeq$ $\left(\begin{array}{cc}S^{*} & M \\ 0 & T\end{array}\right)$, where $S$ is a left filial algebra such that $S=l_{S}(\mathcal{R}(S))+\mathcal{R}(S)$ and $\mathcal{R}(S) \neq 0, T$ is a strongly regular algebra, $M$ is an $S^{*}-T$-bimodule, which is unitary as the left $S^{*}$-module and such that $S M=0$.

Proof. Suppose that $A$ satisfies Theorem 4.3 (ii). It is clear that $e A e \simeq S^{*}$, where $S=e l_{A}(\mathcal{R}(A)) e+e \mathcal{R}(A) e$. Note that $((1-e) A e)^{2}=0$. Hence, since $A$ is left filial, $(1-e) A e \subseteq \mathcal{R}(A)$. Now for every $b \in \mathcal{R}(A), e b=b$ and $e(1-e) A=0$, so $((1-e) A) \cap \mathcal{R}(A)=0$. In particular $(1-e) A e=0$ and $(1-e) A(1-e) \cap \mathcal{R}(A)=0$. Set $M=e A(1-e)$. Clearly $M^{2}=0$, so $M \subseteq \mathcal{R}(A)$ and by Theorem 3.3, $\mathcal{R}(A) M=0$. This implies that $S M=0$. It is not hard to check that $\mathcal{R}(e A e)=e \mathcal{R}(A) e$, so $A / \mathcal{R}(A) \simeq e(A / \mathcal{R}(A)) e \oplus(1-e) A(1-e)$. Hence $T=(1-e) A(1-e)$ is a strongly regular algebra. It is clear that $M$ is an $S^{*}-T$-bimodule, which is unitary as the left $S^{*}$-module. Now applying the usual Pierce decomposition of $A$ one immediately gets that $A \simeq\left(\begin{array}{c}S^{*} \\ 0\end{array} \frac{M}{T}\right)$.

Conversely, it is not hard to check that $\mathcal{R}\left(\left(\begin{array}{cc}S^{*} & M \\ 0 & T\end{array}\right)\right)=\left(\begin{array}{cc}\mathcal{R}(S) & M \\ 0 & 0\end{array}\right) \simeq \mathcal{R}(S) \oplus M^{0}$, where $M^{0}$ denotes the algebra with zero multiplication on the $F$-space $M$. It is clear

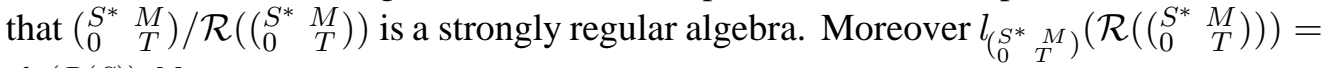
$\left(\begin{array}{cc}l_{S}(\mathcal{R}(S) & M \\ 0 & T\end{array}\right)$, so $\left(\begin{array}{cc}S^{*} & M \\ 0 & T\end{array}\right)=F\left(\begin{array}{ll}1 & 0 \\ 0 & 0\end{array}\right)+l_{\left(\begin{array}{c}S^{*} \\ 0\end{array}\right)}\left(\mathcal{R}\left(\left(\begin{array}{cc}S^{*} & M \\ 0 & T\end{array}\right)\right)\right)+\mathcal{R}\left(\left(\begin{array}{cc}S^{*} & M \\ 0 & T\end{array}\right)\right)$ and $\left(\begin{array}{cc}S^{*} M \\ 0 & T\end{array}\right)$ is of the form described in Theorem 4.3 (ii).

We can get a more precise description of algebras satisfying Theorem 4.3 when $A / \mathcal{R}(A)$ is a finite dimensional algebra.

Let $T$ an algebra with identity and $M$ a unitary right $T$-module. The set $\left(\begin{array}{ll}T & 0 \\ M & 0\end{array}\right)$ of matrices of the form $\left(\begin{array}{cc}t & 0 \\ m & 0\end{array}\right)$, where $t \in T$ and $m \in M$, is an algebra with respect to obvious matrix operations.

Theorem 4.5. An algebra $A$ such that $\operatorname{dim}_{F}(A / \mathcal{R}(A))<\infty$ satisfies Theorem 4.3 (i) if and only if $A \simeq\left(\begin{array}{cc}T & 0 \\ M & 0\end{array}\right) \oplus B$, where $T$ is a finite dimensional strongly regular algebra with identity, $M$ is a unitary right $T$-module and $B$ is a nilpotent left filial algebra. 
Proof. Suppose that $A$ is an algebra satisfying Theorem 4.3 (i) and such that $A / \mathcal{R}(A)<\infty$. Since $A$ is left filial, $(\mathcal{R}(A))^{3}=0$. By the assumption $A=l_{A}(\mathcal{R}(A))+\mathcal{R}(A)$. These imply that $A^{2} \mathcal{R}(A)=0$.

Since $\operatorname{dim}_{F} A / \mathcal{R}(A)<\infty$ and $A / \mathcal{R}(A)$ is strongly regular it has an identity, which can be lifted to an idempotent $e$ of $A$. Then $A=e A+\mathcal{R}(A)$. Note that if $e x \in e A \cap \mathcal{R}(A)$, then, since $e \mathcal{R}(A)=e^{2} \mathcal{R}(A) \subseteq A^{2} \mathcal{R}(A)=0$ and $e(e x)=e x$, we get that $e x=0$. Thus $e A \cap \mathcal{R}(A)=0$. This in particular shows that $e A$ is a strongly regular algebra. Since idempotents in reduced algebras are central, $e$ is the identity element of $e A$. In particular $e A=e A e$. Obviously $B=\{r-r e \mid r \in \mathcal{R}(A)\}$ is a subalgebra of $A$ contained in $\mathcal{R}(A)$. Hence, since $A$ is a left filial algebra, $B \triangleleft \mathcal{R}(A)$. Moreover $e A B=B e A=0$. Hence $B \triangleleft A$. One easily checks that $e A+\mathcal{R}(A) e \triangleleft A$. Obviously $(e A+\mathcal{R}(A) e) \cap B=0$ and $A=e A+\mathcal{R}(A) e+B$. Hence $A=(e A+\mathcal{R}(A) e) \oplus B$. This in particular shows that $M=e \mathcal{R}(A)$ is a unitary right $T$-module, where $T=e A$. Now it is clear that $A \simeq\left(\begin{array}{ll}T & 0 \\ M & 0\end{array}\right) \oplus B$. This proves the "only if" part of the theorem. The "if" part is obvious.

We do not know whether Theorem 4.5 can be extended to all algebras. One can prove that it would be so if every algebra $A$ such that $A \mathcal{R}(A)=0$ and $A / \mathcal{R}(A)$ is strongly regular, contained a right ideal $I$ such that $I \cap \mathcal{R}(A)=0$ and $A=I+\mathcal{R}(A)$.

Combining Theorems 4.3, 4.4, 4.5 and the results obtained in Section 3 it is routine to get a complete classification of finite dimensional left filial algebras for fields over which finite dimensional prime radical $H$-algebras are classified, in particular for algebraically closed and finite fields.

\section{The Structure of Algebras which are Filial and Left Filial}

As we have seen earlier semiprime left filial algebras are filial and prime radical algebras are left filial if and only if they are filial. There are semiprime filial algebras (e.g., the algebra of $2 \times 2$-matrices over $F$ ), which are not left filial. The following example shows that, in general, left filial algebras need not be filial.

Example. Let $A=F\left(e_{11}+e_{22}\right)+F\left(e_{12}+e_{21}\right)+F e_{31}+F e_{32}$, where $e_{i j}$ denote the standard matrix units of the algebra $M_{3}(F)$ of $3 \times 3$-matrices over $F$ with $c h F \neq 2$. It is not hard to see that $A$ is a subalgebra of $M_{3}(F), \mathcal{R}(A)=$ $F e_{31}+F e_{32}, A / \mathcal{R}(A) \simeq F \oplus F$ and $A \mathcal{R}(A)=0$. Hence by Theorem 4.3, $A$ is left filial. Now $F e_{31} \triangleleft F e_{31}+F e_{32}=\mathcal{R}(A) \triangleleft A$. However $e_{31}\left(e_{12}+e_{21}\right)=e_{32}$, so $F e_{31} \Varangle A$. Hence $A$ is not filial.

It is clear that left and right filial algebras are also filial. It turns out that arbitrary two of these conditions imply the third.

Theorem 5.1. Every algebra A, which is left filial and filial is also right filial. 
Proof. Suppose that $K<_{r} I<_{r} A$. We have to show that $K<_{r} A$. Obviously $K \cap \mathcal{R}(A)$ is a subalgebra of $\mathcal{R}(A)$ and $\mathcal{R}(A)$ is a left filial algebra. Hence by Theorem 3.3, $K \cap \mathcal{R}(A) \triangleleft \mathcal{R}(A)$ and, since $A$ is a filial algebra, $K \cap \mathcal{R}(A) \triangleleft A$. Factoring out $K \cap \mathcal{R}(A)$, we can assume that $K \cap \mathcal{R}(A)=0$. Since $A / \mathcal{R}(A)$ is a semiprime left filial algebra, we get that $K \simeq(K+\mathcal{R}(A)) / \mathcal{R}(A)$ is an idempotent algebra. Hence $K A=K^{2} A=K(K A) \subseteq K I \subseteq K$, so $K<_{r} A$.

Dual arguments show that every algebra, which is right filial and filial is also left filial.

It is well known and not hard to check that the class of strongly regular algebras is a radical class. For a given algebra $A$ we denote by $\mathcal{S}(A)$ the strongly regular radical of $A$, i.e., the largest strongly regular ideal of $A$.

Now we describe the structure of algebras, which are left filial and filial. We start with the following proposition.

Proposition 5.2. If $A$ is an algebra such that $A \mathcal{R}(A)=0=\mathcal{R}(A) A$ and $A / \mathcal{R}(A)$ is strongly regular, then $A=\mathcal{S}(A) \oplus \mathcal{R}(A)$.

Proof. It is clear that $\mathcal{S}(A) \cap \mathcal{R}(A)=0$. Take any $a \in A$. Since $A / \mathcal{R}(A)$ is strongly regular, there is an idempotent $t \in A / \mathcal{R}(A)$ such that $(a A+\mathcal{R}(A)) / \mathcal{R}(A)=$ $t(A / \mathcal{R}(A))$. Lifting $t$ to an idempotent $e$ of $A$ we get that $a A+\mathcal{R}(A)=e A+\mathcal{R}(A)$. If for some $x \in A$, ex $\in \mathcal{R}(A)$, then $e x=e(e x) \in A \mathcal{R}(A)=0$, so $e A \cap \mathcal{R}(A)=0$. Since $A / \mathcal{R}(A)$ is strongly regular, so is $e A$ and $(e A+\mathcal{R}(A)) / \mathcal{R}(A) \triangleleft A / \mathcal{R}(A)$. Thus $A(e A) \subseteq e A+\mathcal{R}(A)$ and $A(e A)=A(e A)^{2} \subseteq(e A+\mathcal{R}(A)) e A \subseteq e A+$ $\mathcal{R}(A) A=e A$. These show that $e A \triangleleft A$, so $e A \subseteq \mathcal{S}(A)$. Consequently $a \in$ $e A+\mathcal{R}(A) \subseteq \mathcal{S}(A)+\mathcal{R}(A)$. Hence $A=\mathcal{S}(A) \oplus \mathcal{R}(A)$.

The following theorem describes the structure of left and right filial algebras (equivalently, filial and left (right) filial algebras).

Theorem 5.3. An algebra $A$ is left and right filial if and only if $\mathcal{R}(A)$ is an $H$-algebra and one of the following cases holds

(i) $A=\mathcal{S}(A) \oplus \mathcal{R}(A)$;

(ii) $A=F f+(\mathcal{S}(A) \oplus \mathcal{R}(A))$, where $\mathcal{R}(A) \neq 0$ and $f$ is an idempotent of $A$ such that $f b=0$ and $b f=b$ for every $b \in \mathcal{R}(A)$;

(iii) $A=F e+(\mathcal{S}(A) \oplus \mathcal{R}(A))$, where $\mathcal{R}(A) \neq 0$ and $e$ is an idempotent of $A$ such that $e b=b$ and $b e=0$ for every $b \in \mathcal{R}(A)$;

(iv) $A=F e+F f+(\mathcal{S}(A) \oplus \mathcal{R}(A))$, where $\mathcal{R}(A) \neq 0$ and e, $f$ are idempotents of $A$ such that $e b=b$, be $=0$ and $f b=0, b f=b$ for every $b \in \mathcal{R}(A)$. 
Proof. Suppose that $A$ is left and right filial. Set $R=\mathcal{R}(A)$ and $I=l_{A}(R)$. Obviously $I \triangleleft A$ and $\mathcal{R}(I)=I \cap \mathcal{R}(A)$.

By Theorem 4.3, $R$ is an $H$-algebra and $A / R$ is strongly regular. Moreover

(a) $A=I+R$

or

(b) $A=F e+I+R$, where $R \neq 0$ and $e$ is an idempotent of $A$ such that $e b=b$ for every $b \in R$.

Suppose that a) holds. Obviously $\mathcal{S}(I) \subseteq \mathcal{S}(A)$. On the other hand $\mathcal{S}(A)=$ $(\mathcal{S}(A))^{3} \subseteq(I+R)^{3} \subseteq I+R^{3}=I$. Hence $\mathcal{S}(A)=\mathcal{S}(I)$. If $\mathcal{R}(I) I=0$, then by Proposition 5.2, $I=\mathcal{S}(I) \oplus \mathcal{R}(I)$. Then $A=I+R=\mathcal{S}(I)+\mathcal{R}(I)+R=\mathcal{S}(A)+R$ and, since $\mathcal{S}(A) \cap R=0$, we get that $A=\mathcal{S}(A) \oplus R$, so (i) holds. Suppose now that $\mathcal{R}(I) I \neq 0$. Then applying the dual version of Theorem 4.3 and Proposition 5.2 we get that $I=F f+(\mathcal{S}(I) \oplus \mathcal{R}(I))$ for an idempotent $f$ of $I$ such that $b f=b$ for every $b \in \mathcal{R}(I)$. The dual version of Lemma 4.1 implies that also $b f=b$ for every $b \in R$. Now $A=I+R=F f+\mathcal{S}(I)+R=F f+\mathcal{S}(A)+R$ and, since $\mathcal{S}(A) \cap R=0$, we get $A=F f+(\mathcal{S}(A) \oplus R)$. Thus (ii) follows.

Suppose now that b) holds. As we have seen above $\mathcal{S}(I+R)=\mathcal{S}(I)$. Now for every $r \in R$ such that $r^{2}=0,(I+R) r=0$. Hence if for some $\alpha \in F$ and $t \in I+R, u=\alpha e+t \in \mathcal{S}(A)$, then $\alpha r=\alpha e r=u r \in \mathcal{S}(A) \cap R=0$. These show that $\mathcal{S}(A)=\mathcal{S}(I+R)=\mathcal{S}(I)$. Now applying to $I+R$ the arguments used in the proof in case a) one obtains that $A$ satisfies (iii) or (iv).

If $A$ satisfies (i), (ii) or (iii), then Theorem 4.3 or its dual version immediately imply that $A$ is left and right filial. If $A$ satisfies (iv), then $l_{A}(R) \supseteq F f+\mathcal{S}(A)$ and $e b=b$ for every $b \in R$. Hence by Theorem 4.3, $A$ is left filial. Next, $r_{A}(R) \supseteq F e+\mathcal{S}(A)$ and $b f=b$ for every $b \in R$. Hence the dual version of Theorem 4.3 gives that $A$ is right filial. The result follows.

Theorem 5.3 reduces a classification of left filial algebras to classifications of strongly regular algebras and prime radical $H$-algebras.

\section{REFERENCES}

1. R. R. Andruszkiewicz, The classification of integral domains in which the relation of being an ideal is transitive, Comm. Algebra, 31 (2003), 2067-2093.

2. R. R. Andruszkiewicz and E. R. Puczyłowski, On filial rings, Portugaliae Math., 45 (1988), 139-149.

3. G. Ehrlich, Filial rings, Portugaliae Math., 42 (1983/84), 185-194.

4. M. Filipowicz and E. R. Puczyłowski, Left filial rings, Algebra Colloq., 11 (2004), 335-344. 
5. M. Filipowicz and E. R. Puczylowski, On filial and left filial rings, Publ. Math. Debrecen, 66 (2005), 257-267.

6. M. Filipowicz and E. R. Puczyłowski, On the upper radical determined by filial rings, Acta Math. Hungar., 112 (2006), 227-236.

7. K. R. Goodearl, Von Neumann regular rings, Monographs and Studies in Mathematics, Pitman, London, 1979.

8. Liu Shao-xue, On algebras in which every subalgebra is an ideal, Acta Math. Sinica, 14, $532-537$ (Chinese); translated as Chinese Math.-Acta, 5 (1964), 571-577.

9. G. L. Kruse, Rings in which all subrings are ideals, Canad. J. Math., 20 (1968), 862-871.

10. O. T. O'Meara, Introduction to quadratic forms, Classics in Mathematics, SpringerVerlag, Berlin, 2000.

11. A. D. Sands, On ideals in over-rings, Publ. Math. Debrecen, 35 (1988), 273-279.

12. G. Tzintzis, An almost subidempotent radical property, Acta Math. Hung., 49 (1987), 173-184.

13. G. Tzintzis, A one-sided admissible ideal radical which is almost subidempotent, Acta Math. Hung., 49 (1987), 307-314.

14. S. Veldsman, Extensions and ideals of rings, Publ. Math. Debrecen, 38 (1991), 297-309.

\author{
M. Filipowicz \\ Department of Mathematics, \\ Technical University of Białystok, \\ 15-950 Białystok, \\ Wiejska 45 A, Poland \\ E-mail: mfilipowicz@kki.net.pl \\ E. R. Puczyłowski \\ Institute of Mathematics, \\ University of Warsaw, \\ 02-097 Warsaw, \\ Banacha 2, Poland \\ E-mail: edmundp@mimuw.edu.pl
}

